\title{
Immobilization of Radionuclides Through Anaerobic Bio-oxidation of Fe(II)
}

\author{
Coates, John D. \\ University of California, Berkeley
}

\begin{abstract}
RESULTS TO DATE: Over the last year we have focused our efforts on two independent aspects (a) further investigation of the microbiology and geochemistry of nitrate-dependent Fe(II) oxidation and (b) assembling the sequenced genome of Dechloromonas aromatica strain RCB. This work has been performed in a cooperative fashion amongst the independent labs of the three PI?s with the UC Berkeley lab taking the lead under the guidance of J.D. Coates.
\end{abstract}

Nitrate-dependent $\mathrm{Fe}(\mathrm{II})$ oxidation. In an effort to elucidate this microbial metabolic process in the context of an environmental system, a freshwater sediment core was collected and geochemically characterized concurrent with the enumeration of the nitrate-dependent Fe(II)-oxidizing microbial community and isolation of a nitrate-dependent Fe(II)-oxidizing microorganism. Geochemical analysis revealed the presence of both $\mathrm{Fe}(\mathrm{II})$ and nitrate in the upper fourteen centimeters of the sediment core. Most probable number (MPN) enumeration studies revealed the presence of an abundant nitrate-dependent $\mathrm{Fe}$ (II)oxidizing microbial community concurrent with prevailing Fe(II) and NO3- concentrations.. The potential for anaerobic Fe(II) bio-oxidation is supported by MPN enumeration studies which revealed a nitratedependent Fe(II)-oxidizing population as high as 1.47 × 104 cells.g-1 sediment. Similarly, enumeration studies performed using samples collected from several locations across the FRC indicated the presence of a significant indigenous nitrate-dependent Fe(II)-oxidizing microbial community of $2.04 \times 103$ cells mL1 in groundwater, and $1.17 \times 103$ cells (g sediment)- 1 in subsurface sediment.

Cosmobacter millennium strain 2002, a novel autotrophic nitrate-dependent Fe(II)-oxidizer During these studies a novel autotrophic nitrate-dependent Fe(II)-oxidizing organism, strain 2002, was isolated. Optimal growth was observed under low salt conditions, $0 \%$ salinity, of basal culture media, $\mathrm{pH} 7.25$, at a temperature of $37 \mathrm{C}$. The DNA G+C content was $63.8 \mathrm{~mol} \%$. Phylogenetic analysis of the 16S rRNA gene sequence placed strain 2002 in the order Neisseriales within the beta subclass of the Proteobacteria with 95.1\% similarity to Chromobacterium suttsuga. In contrast to Chromobacterium sp., strain 2002 does not produce violacein or cyanide nor is it capable of fermentative growth or fumarate reduction.

Chromobacterium species not previously recognized for the ability of nitrate-dependent Fe(II) oxidation. Under non-growth conditions both strain 2002 and C. violaceum incompletely reduced nitrate to nitrite with $\mathrm{Fe}(\mathrm{II})$ as the sole electron donor. In contrast, strain 2002 reduced nitrate to gaseous end-products under growth conditions with $\mathrm{Fe}(\mathrm{II})$ as the electron donor. Lithoautotrophy was verified by the requirement of $\mathrm{CO} 2$ for growth under nitrate-dependent Fe(II)-oxidizing conditions and by the assimilation of $14 \mathrm{C}$ into organic carbon when the cells were grown in 14CO2. Form I or II Rubisco was not observed in strain 2002 in a screen for ribulose-1,5 biphosphate carboxylase, the enzyme responsible for $\mathrm{CO} 2$ fixation in the Calvin Cycle. The results of these studies provide the first example of an anaerobic, mesophilic, neutrophilic $\mathrm{Fe}(\mathrm{II})$-oxidizing lithoautotroph isolated from a freshwater environment. On the basis of phenotypic and phylogenetic characterization, a new genus Cosmobacter is proposed for this organism with Cosmobacter millennium strain 2002 as the type strain. In addition to Cosmobacter millennium strain 2002, two other nitrate-dependent Fe(II)-oxidizers were also obtained from enrichment cultures one of which was closely related to Azospira (formerly Dechlorosoma) suillium strain PS which we previously demonstrated as a ubiquitous nitrate-dependent Fe(II)-oxidizer in the environment. Nitrate-dependent metal oxidation by A. suillum was specific for $\mathrm{Fe}$ (II). In an effort to identify proteins associated with $\mathrm{Fe}$ (II) oxidation in A. suillum we investigated the expressed protein content of cells grown under NO3-reducing conditions in the presence and absence of Fe(II). Electrophoresis of the total protein content of cell lysates prepared from these cultures did not reveal the selective expression of any proteins associated with the presence of $\mathrm{Fe}(\mathrm{II})$. Similar results were obtained when electrophoresed proteins were stained 
with o-dianisidine based heme stain to identify cytochromes specifically associated with this metabolism. Endproducts of $\mathrm{Fe}$ (II) oxidation Previously we demonstrated that nitrate-dependent bio-oxidation of $\mathrm{Fe}$ (II) by Azospira suillium strain PS results in the formation of crystalline mixed $\mathrm{Fe}(\mathrm{II}) / \mathrm{Fe}(\mathrm{III})$ mineral phases which subsequently immobilize heavy metals and radionuclides. Similarly to A. suillium strain PS, Cosmobacter millennium strain 2002, yields a mixed phase $\mathrm{Fe}(\mathrm{II} / \mathrm{Fe}(\mathrm{III})$ mineral phase, identified as green rust by Mossbauer spectroscopy and X-Ray diffraction. Green rust (GR), a mixed valence, layered $\mathrm{Fe}(\mathrm{II} / \mathrm{Fe}(\mathrm{III})$ hydroxide with anion interlayers, is abundant in hydropmorphic soils and sediments. Recent studies suggested that both Fe(II)-oxidizing and Fe(III)-reducing bacteria play a role in GR formation, however, the extent of either process is unknown. In the case of Fe(III)-reduction, GR is transiently produced and subsequently transformed into magnetite. Anaerobic nitrate-dependent oxidation of Fe(II) provides an alternative mechanism of GR formation. In contrast to the GR biogenically formed by Fe(III) reduction, the biogenic GR product formed via nitrate-dependent $\mathrm{Fe}(\mathrm{II})$ oxidation by $\mathrm{C}$. millennium strain 2002 did not yield transformation products, i.e., magnetite. X-Ray fluorescence spectroscopy identified chloride and phosphate in association with the GR product. Furthermore, anion analysis of the GR by ion chromatography indicated that $6 \mathrm{mg} \mathrm{SO} 42-, 32 \mathrm{mg} \mathrm{Cl}$-, and $66 \mathrm{mg} \mathrm{PO} 43-$ was associated with the oxidation of $111 \mathrm{mg} \mathrm{Fe}(\mathrm{II})$. These results suggest that $\mathrm{GR}(\mathrm{Cl}-1)$ was predominantly formed. It is currently unknown whether phosphate is adsorped to the GR surface or intercalated into the interlayer. Given that nitrate-dependent $\mathrm{Fe}$ (II) oxidizing bacteria are ubiquitous, as identified by most probable number enumeration studies outlined above, it is likely that this metabolism is active in soils and sedimentary environments These results represent the first demonstration of the biogenic formation of green rust in significant quantities providing strong evidence for the biological mechanism for the production of $\mathrm{GR}(\mathrm{Cl}-)$ in soils and sediments. Further investigation of the biogenic iron mineral phases produced by $A$. suillum in the presence of $100 \mathrm{mM} \mathrm{U}(\mathrm{VI})$ were analyzed by density gradient centrifugation resulting in the recovery of five distinct fractions. Differential solubility analysis of $0.5 \mathrm{M} \mathrm{HCl}$ extractable $\mathrm{Fe}$ and $3 \mathrm{M} \mathrm{HCl}$ extractable Fe of each of these fractions indicated the existence of a positive correlation between fraction density and crystallinity. Percent of $0.5 \mathrm{M} \mathrm{HCl}$-extractable Fe decreased from $100 \%$ to $3 \%$ with an increase in sucrose concentration (20 to 60\%) indicating separation of crystalline Fe compounds. Analysis of the total iron content in each fraction revealed that most of the iron $(>86 \%)$ was present in the more dense crystalline phases. Similarly, analysis of the $\mathrm{U}(\mathrm{VI})$ content revealed that the majority $(\sim 80 \%)$ of the $\mathrm{U}(\mathrm{VI})$ was also associated with the most dense crystalline phase $(3 \% 0.5 \mathrm{M} \mathrm{HCl}$ extractable). The ratio of $3 \mathrm{M} \mathrm{HCl}$-extractable $\mathrm{Fe}(\mathrm{II})$ to total $\mathrm{Fe}(0.68)$ was also highest in this fraction suggesting crystalline mixed $\mathrm{Fe}(\mathrm{II}) / \mathrm{Fe}(\mathrm{III})$ phases. The formation of a mixed phase crystalline Fe compound sequestering a significant proportion of $\mathrm{U}(\mathrm{VI})$ is an ideal remediation strategy as this compound associated with the $\mathrm{U}(\mathrm{VI})$ would not be easily re-mobilized by reductive dissolution of Fe. These results demonstrate that biogenic crystalline Fe compounds can sequester significant proportions of HMR $(\mathrm{U}(\mathrm{VI}))$. Furthermore, existing insitu geochemical conditions for microbial anaerobic Fe(II) oxidation and the presence of microorganisms capable of this metabolism supports the applicability of this remediation strategy for the insitu sequestration of HMR on a long-term basis. Completed Genome Sequence of Dechloromonas aromatica strain RCB. Dechloromonas aromatica strain RCB is a beta class proteobacterium found ubiquitously in soil and sediment environments. It is a motile facultative anaerobe, capable of nitratedependent Fe(II) oxidation, anaerobic aromatic hydrocarbon degradation, and dissimilatory perchlorate reduction. It was first isolated from Potomac River sediments based on its ability to anaerobically metabolize 4-chlorobenzoate coupled to perchlorate reduction. With its metabolic versatility, D. aromatica strain RCB was selected for complete genome sequencing at the DOE Joint Genome Institute, Walnut Creek, CA. The now completed sequence consists of a single circular chromosomal DNA structure containing a total of $4.5 \mathrm{Mb}$ of DNA with a G+C content of $60 \%$. Initial draft annotation was conducted at Oak Ridge National Laboratories and the Virtual Institute for Microbial Stress and Survival (http://escalante.lbl.gov) identifying approximately 4000 open reading frames (ORFs). The closest relative with a completed genome sequence is Ralstonia solanacearum, also a gram negative proteobacteria of the Beta sub-class. D. aromatica and Ralstonia possess very similar genomic content, duplicative $23 \mathrm{~S}$ and 16S RNA regions, and identical operon structures for many families of genes. In Ralstonia, many catabolic capabilities are thought to be concentrated within plasmid DNA structures. However, D. aromatica lacks a large genomic plasmid element (the Ralstonia pGMI1000MP plasmid is $2.09 \mathrm{Mb}$ in size), which indicates its metabolic versatility is not dependent on a highly transmissible and mutable plasmid structure. D. aromatica genes have been annotated for InterPro domains, EC assignments, TIGRfams, KEGGS pathways, and GO ontologies. Genes that likely reflect the versatility of $D$. aromatica 
within the environment include a remarkably high number of two-component sensors and regulators (11\% of the annotated ORFs), placing it in the top $5 \%$ of microbial genomes in this capability. The D. aromatica genome has been found to contain RuBisCo, indicating the ability to fix carbon dioxide, however, to date the environmental conditions required for the expression of this phenotype have not been identified. The availability of the completed and partially annotated genome sequence provides us the opportunity to design and utilize a custom D. aromatica microarray to identify genes that are upregulated under Fe(II)oxidizing conditions and, thus, presumably involved in this anaerobic metabolism.

DELIVERABLES: 1 . Cummings, D.E., O.L. Snoeyenbos-West, D.T. Newby, A.M. Niggemyer, D.R. Lovley, L.A. Achenbach, and R.F. Rosenzweig. 2003. Diversity of Geobacteraceae species inhabiting metal-polluted freshwater lake sediments ascertained by 16S rDNA analyses. Micro. Ecol. (in press). 2. Coates, J.D., Chakraborty, R., McInerney, M.J. (2002). Anaerobic benzene biodegradation - a new era. Research in Microbiology 153:621-628 3. Coates, J.D. and Chakraborty, R. (2002). Anaerobic bioremediation - an emerging resource for environmental cleanup. In Singleton, I., Milner, M.G., and Head, I.M. "Bioremediation: a critical review" Horizon Press, Norfolk, UK pp 227-259. 4. Lack, J.G., Chaudhuri, S.K., Chakraborty, R., Achenbach, L.A., and Coates, J.D. (2002). Anaerobic biooxidation of $\mathrm{Fe}(\mathrm{II})$ by Dechlorosoma suillum. Microbial Ecology 43:424-431. 5. Lack, J.G., Chaudhuri, S.K., Kelly, S.D., Kemner, K.M., O'Connor, S.M., and Coates, J.D. (2002). Immobilization of radionuclides and heavy metals through anaerobic biooxidation of Fe(II). Applied and Environmental Microbiology 68:2704-2710. 6. Coates, J.D. (2002). Bacteria that Respire Oxyanions of Chlorine. In Brenner, Krieg, Staley and Garrity (Editors) Bergey's Manual of Systematic Bacteriology, 2nd Edition, Volume 2, Springer-Verlag, New York, NY (in press). 